\title{
Comparison of balance and out of balance main battle tank armaments
}

\author{
David J. Purdy \\ Royal Military College of Science, Shrivenham \\ Swindon England, SN6 8LA
}

It has been commonly thought that stabilising an out of balance gun on a moving platform (tank or ship) is very difficult or impossible to achieve. Using models of a balanced and out of balance gun on a main battle tank this is shown not to be the case. The models of the guns used, include the effect of non-linear friction and out of balance. To improve the stabilisation of the out of balance gun, trunnion vertical acceleration feedforward is used.

\section{Introduction}

The primary objective of the Weapon Control System (WCS) on a Main Battle Tank (MBT) is to maximise the probability of hitting a stationary or moving target with the first round, in the shortest possible time, from a stationary or moving vehicle. The current practice for the designers of tanks is to place the centre of gravity at the trunnions. This is because of the widely held belief that if the centre of gravity is not aligned with the trunnion the stabilisation of the gun will be significantly reduced. Two quotes from the literature relating to this are:

"With this equilibrating system a complete equilibrium can only be achieved for a stationary gun on a horizontal base. If the gun is tipped on its trunnion axis on sloping ground, or, as with tank and naval guns, rocked while travelling, then additional mass forces on the center of gravity of the elevating part cause the compensation to be disrupted.

For this reason, the naval guns [and presumably tank guns] can not use an equilibrator and must place the center of gravity of the elevating part in the trunnion axis." [1],

and

"An equilibrator can only balance an otherwise unbalanced system statically. It will be apparent that, in the dynamic state, acceleration forces act at the centre of gravity of the elevating mass, giving rise to varying moments that cannot be counteracted by a conventional equilibrator. It is for this reason that equilibrators cannot be used in stabilised systems." [2].

The current mounting practice for the main armament in MBTs [1-3] is to support the gun in a cradle, which allows the gun to recoil when fired. The high acceleration of the gun during recoil prevents the easy attachment of sensors to it. Thus the motions of the cradle are sensed and used for controlling the gun by the WCS. Therefore it is the breech of the gun, which is controlled in current MBTs. Possibilities of controlling the muzzle of the gun have been examined in [5] but in this paper only the motions of the breech will be considered.

Using simulation techniques, this paper, compares the quality of stabilisation of the elevation axis for two WCS, one having a balanced gun and the other an Out Of Balance (OOB) gun. The elevation model and closed-loop controller used in this study have been taken from $[3,4]$. The elevation model can be either linear or non-linear, the principal non-linearities in this model are the OOB, and static and kinetic friction. The elevation model allows the MBT motions to be coupled into the gun via the hull pitch rate and vertical acceleration at the trunnions. The gun barrel is modelled as two rigid sections and is referred to in the paper as a Lumped Parameter Flexible Beam Model (LPFBM). To improve the stabilisation of the OOB gun, trunnion vertical acceleration feedforward is incorporated into the controller.

\section{Weapon control system models}

A brief description of the models used is given in this section, the interested reader is referred to [3,5] for a more detailed description of the models. This section is broken down into three subsections, the first two subsections examine the elevation models for the balanced and OOB guns respectively and the third subsection shows the simulated results of the two elevation models. 


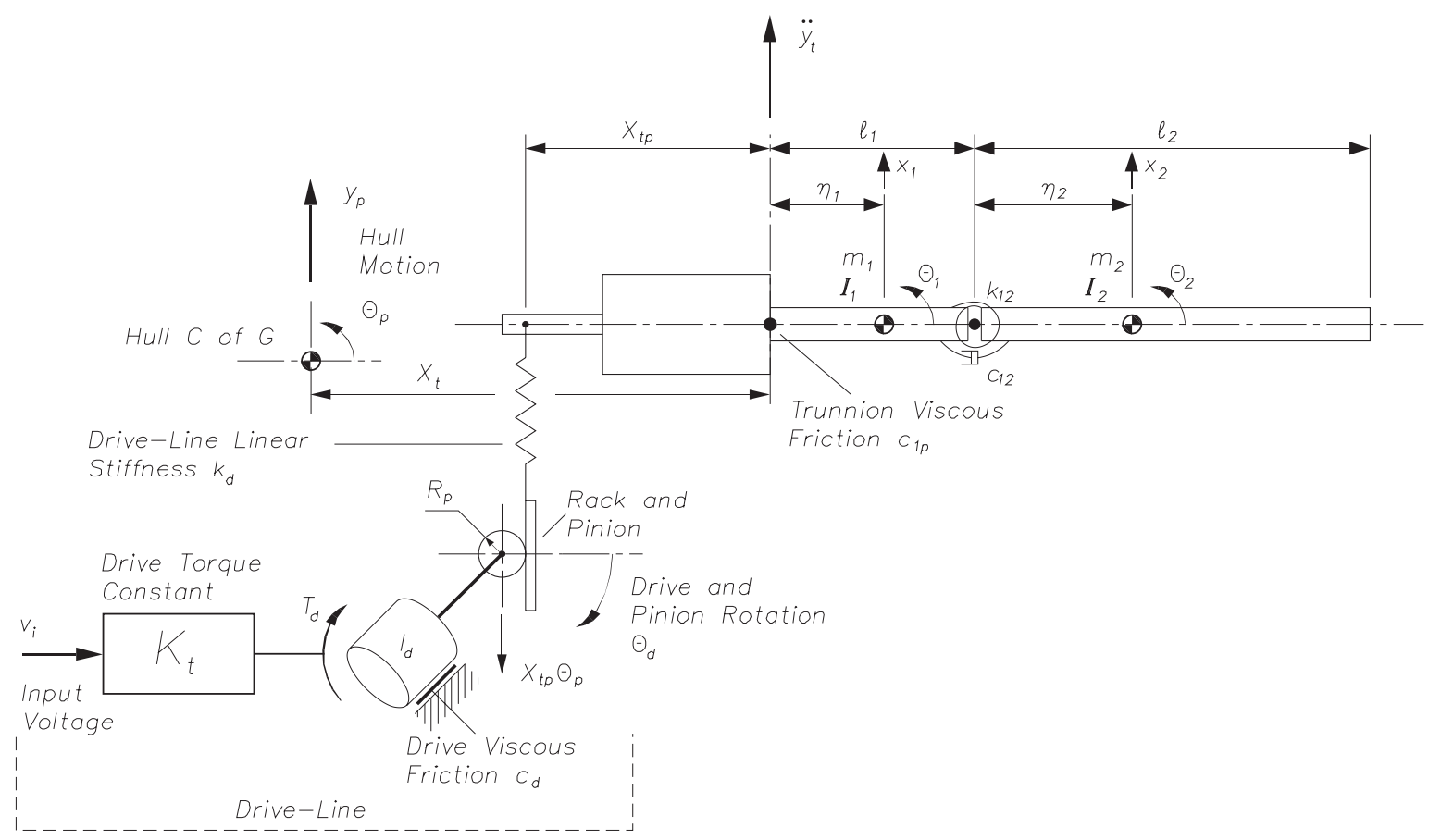

Fig. 1. Elevation model with two section LPFBM.

\subsection{Balanced gun}

A diagram of the elevation channel is shown in Fig. 1. The input to the elevation drive is a voltage to the servoamplifier. The servo-amplifier produces a current, proportional to its input voltage. The prime mover is a d.c. servo-motor and in conjunction with the amplifier, can be considered as producing torque proportional to its input current [6]. The remainder of the drive-line consists of a gearbox, and rack and pinion. The servoamplifier, motor and gearbox are represented by a single drive torque constant $K_{t}$. Sensors are used to measure the angular rate of the motor, and angular rate and position of the cradle.

The drive inertia $I_{d}$, represents the motor inertia $I_{m}$ referred to the output of the gearbox of ratio $G$ and is given by:

$$
I_{d}=I_{m} G^{2}
$$

and the drive torque by:

$$
T_{d}=K_{t} v_{t}
$$

where $v_{i}$ is the input to the servo-amplifier. The viscous friction at the drive is $c_{d}$ and the radius of the pinion is $R_{p}$. The drive-line stiffness $k_{d}$ has been lumped between the rack and the cradle, which is equivalent to the model given in $[5,6]$. The cradle, breech and gun barrel in this model are represented by two rigid sections, of length $l_{1}$ and $l_{2}$, mass $m_{1}$ and $m_{2}$, and moment of inertia about the centre of gravity $I_{1}$ and $I_{2}$. The distance to the centres of gravity are $\eta_{1}$ and $\eta_{2}$, the pin-joint linking the two sections has a torsional stiffness of $k_{12}$ and viscous friction $c_{12}$. This type of flexible beam model has been used to simulate and control flexible space-borne manipulators [7] and to investigate the design of WCSs [3-5]. The method used to select the lengths of the rigid sections is given in [3, 5], in which the muzzle displacement and rotation for the first cantilever mode are matched to a finite element model. The torsional spring rate is calculated to make the first cantilever mode frequencies of the LPFBM and finite element models equal.

The inputs to the model are the voltage to the servoamplifier $v_{i}$, which is the command, the trunnion vertical acceleration $\ddot{y}_{t}$ and the MBT hull pitch rate $\dot{\theta}_{p}$, which are the disturbances. The model responses are the drive angular velocity $\dot{\theta}_{d}$, the breech angle $\theta_{1}$ and velocity $\dot{\theta}_{1}$ and the muzzle angle $\theta_{2}$. The equations of motion for small motions are:

$$
M_{1}\{\ddot{\theta}\}+C_{1}\{\dot{\theta}\}+K_{1}\{\theta\}=I_{1}\{u\}
$$

where the mass $M_{1}$, damping $C_{1}$, stiffness $K_{1}$, and input $I_{1}$ matrices are give by: 


$$
\begin{aligned}
& M_{1}=\left[\begin{array}{ccc}
I_{d} & 0 & 0 \\
0 & I_{1}+m_{1} \eta_{1}^{2}+m_{2} l_{1}^{2} & m_{2} l_{1} \eta_{2} \\
0 & m_{2} l_{1} \eta_{2} & I_{2}+m_{2} \eta_{2}^{2}
\end{array}\right] \\
& C_{1}=\left[\begin{array}{ccc}
c_{d} & 0 & 0 \\
0 & c_{12}+c_{1 p}-c_{12} \\
0 & -c_{12} & c_{12}
\end{array}\right] \\
& K_{1}=\left[\begin{array}{ccc}
k_{d} R_{p}^{2} & -k_{d} R_{p} X_{t p} & 0 \\
-k_{d} R_{p} X_{t p} & k_{12}+k_{d} X_{t p}^{2} & -k_{12} \\
0 & -k_{12} & k_{12}
\end{array}\right] \\
& I_{1}=\left[\begin{array}{cccc}
K_{t} & 0 & 0 & -k_{d} X_{t p} R_{p} \\
0 & -\left(m_{1} \eta_{1}+m_{2} l_{1}\right) & c_{1 p} & k_{d} X_{t p}^{2} \\
0 & -m_{2} \eta_{2} & 0 & 0
\end{array}\right]
\end{aligned}
$$

The vector of inputs and coordinates for the model are:

$$
\begin{aligned}
& \{u\}^{T}=\left\{v_{t} \ddot{y}_{t} \dot{\theta}_{p} \theta_{p}\right\} \\
& \{\theta\}^{T}=\left\{\theta_{d} \theta_{1} \theta_{2}\right\}
\end{aligned}
$$

In state space form the equations for the model states and outputs are given by:

$$
\begin{aligned}
& \dot{x}=A x+B u \\
& y=C x+D u
\end{aligned}
$$

where:

$$
\begin{aligned}
& A=\left[\begin{array}{cc}
0 & I \\
-M_{1}^{-1} K_{1} & -M_{1}^{-1} C_{1}
\end{array}\right] \\
& B=\left[\begin{array}{c}
0 \\
M_{1}^{-1} I_{1}
\end{array}\right] \quad C=\left[\begin{array}{llllll}
0 & 0 & 0 & 1 & 0 & 0 \\
0 & 1 & 0 & 0 & 0 & 0 \\
0 & 0 & 0 & 0 & 1 & 0 \\
0 & 0 & 1 & 0 & 0 & 0
\end{array}\right] \\
& D=\left[\begin{array}{l}
0 \\
0 \\
0 \\
0
\end{array}\right] \\
& \{y\}^{T}=\left\{\dot{\theta}_{d} \theta_{1} \dot{\theta}_{1} \theta_{2}\right\} \\
& \{x\}^{T}=\left\{\theta_{d} \theta_{1} \theta_{2} \dot{\theta}_{d} \dot{\theta}_{1} \dot{\theta}_{2}\right\}
\end{aligned}
$$

and $I$ is a unit matrix of appropriate dimensions.

The non-linear elevation model has been formed by incorporating non-linear friction into the drive and trunnions, and the out-of-balance of the gun. The out-ofbalance torque was caused by the centre of gravity of the elevating mass being $8.0 \mathrm{~mm}$ in front of the trunnions. The non-linear friction model used is a modified reset-integrator representation [8]. The modification to this model includes a random component of friction added into its output. This is generated by integrating white noise and adding it into the friction force, the mean level being zero and the standard deviation being approximately $1 \%$ of the kinetic friction. The static friction provides an additional $25 \%$ of the kinetic friction level. The drive friction was taken as $1 \%$ of the trunnion friction. For the elevation system under investigation the trunnion kinetic friction has been set to $1 \mathrm{kNm}$. A full set of data for the linear and non-linear models and their derivation can be found in [3].

\subsection{Out of balance gun}

The design of the gun and mounting (barrel, breech and cradle) are fixed by considerations beyond the scope of this paper, the interested reader is directed to [1] for further information. Thus the only parameter that can be varied is the position of the trunnions on the cradle, therefore maintaining the dynamics of the gun system. In this paper the trunnions have been moved rearwards, with respect to the centre of mass of the gun and mounting, from its initial value of $0.008 \mathrm{~m}$ to $0.515 \mathrm{~m}$. All the other parameters have remained fixed. The OOB moment generated by this, results in a moment to rotate the breech of the gun $\left(\theta_{1}\right)$ in a negative direction.

The equation for the out of balance moment as a function of breech rotation $\left(\theta_{1}\right)$ is given by:

$$
M_{o b}=-m_{t} g \eta_{t} \cos \theta_{1}
$$

where $M_{o b}$ is the out of balance moment, $m_{t}, g$ and $\eta_{t}$ are the total elevating mass $(2500 \mathrm{~kg})$, acceleration due to gravity $\left(9.81 \mathrm{~m} / \mathrm{s}^{2}\right)$ and the distance from the trunnions to the centre of gravity of the gun system $(0.515 \mathrm{~m})$. The maximum static OOB moment for the gun is $-12.63 \mathrm{kNm}$.

It has been assumed that the static OOB has been totally removed from the gun, by the use of a suitable equilibrator. For a real situation there will still be some residual OOB, which will have a small effect but this has been ignored because it is primarily the OOB that is being considered.

\subsection{Simulated model responses}

To compare the models of the balanced and OOB gun cases, the responses have been plotted in Figs 2 and 3. These plots are for the linear components of the models only. The non-linear elements having been removed.

The responses shown in Fig. 2, are the open-loop frequency responses between the servo-amplifier input 

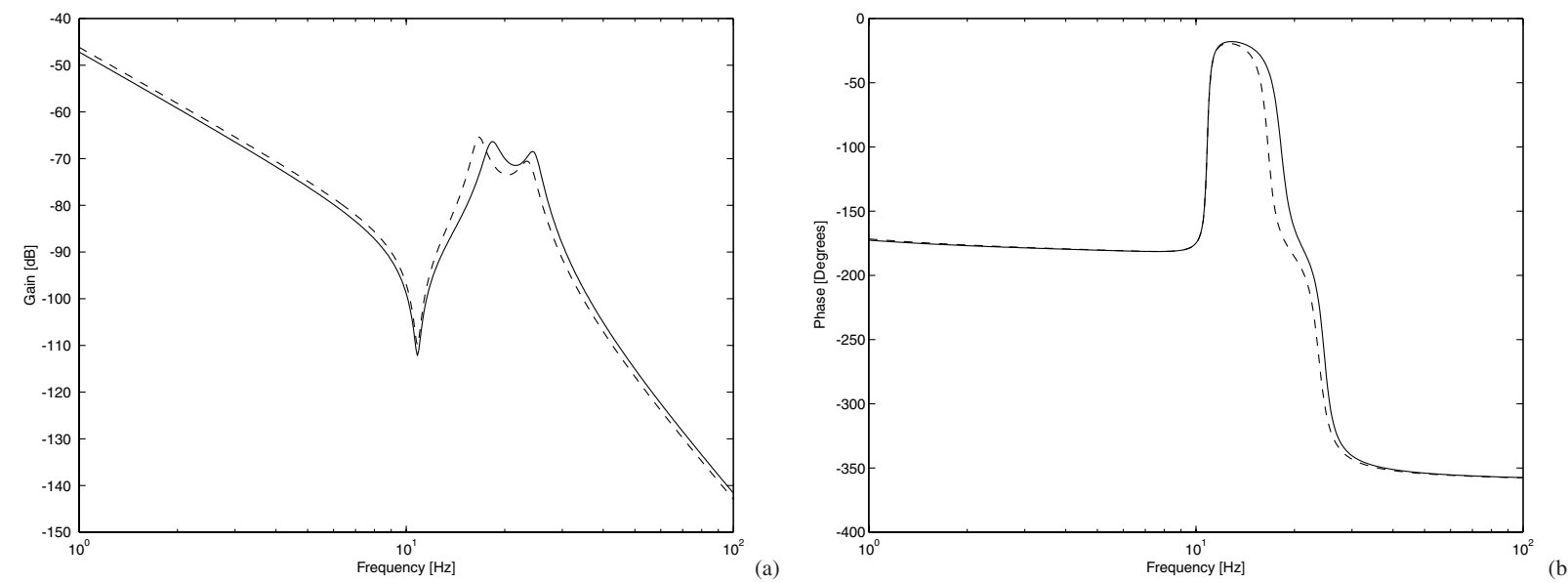

Fig. 2. Plot of the open-loop frequency responses, between the servo-amplifier input and breech angle for the gain (a) and phase (b), with the OOB [solid] and balanced [dash] gun.
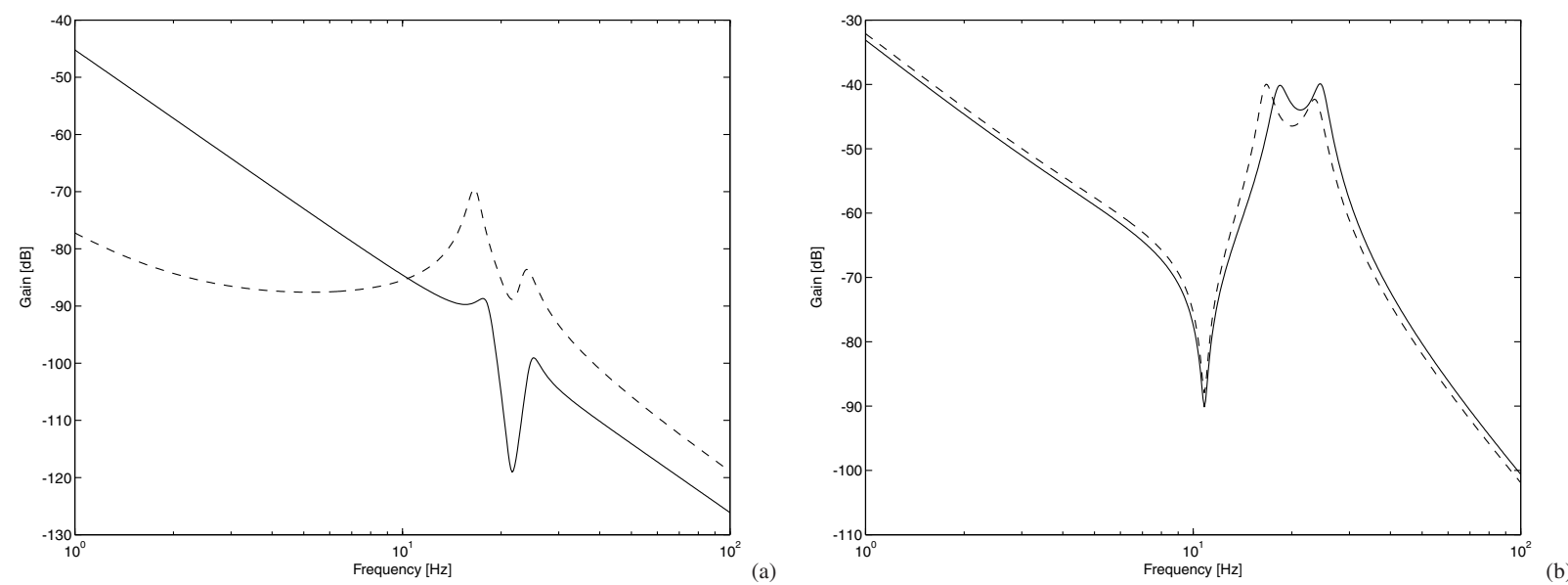

Fig. 3. Plot of the disturbance response of the elevation model breech angle to the trunnion vertical acceleration (a) and hull pitch rate (b), with the OOB [solid] and balanced [dash] gun.

and breech angle. Below $18 \mathrm{~Hz}$ the response of the OOB gun, Fig. 2(a), has a lower gain than the balanced, above $18 \mathrm{~Hz}$ this changes over. This reduction in gain at low frequency is expected because of the increase in inertia. The two resonances (poles) at approximately 18 and $23 \mathrm{~Hz}$, for the balanced gun, have increased in frequency by about $1 \mathrm{~Hz}$ for the OOB case. The anti-resonance (zero) at about $11 \mathrm{~Hz}$ has remained the same, this is to be expected because it represents the first cantilever mode of the barrel [9]. There is very little difference between the phase, Fig. 2(b), for the balanced and OOB situations. The slight differences are due to the changes in the resonances.

The response of the two gun cases to the disturbance inputs are shown in Fig. 3. The response to trunnion vertical acceleration, Fig. 3(a), shows that below
$10 \mathrm{~Hz}$ the OOB gun has a greater gain than the balanced. Above $10 \mathrm{~Hz}$ this changes and the balanced has a greater gain. At $1 \mathrm{~Hz}$ the OOB gun has over $30 \mathrm{~dB}$ greater gain than the balanced. This again is expected, because it is this effect that the authors are referring to in the quotations in the introduction. The point of interest is that at some frequencies the balanced gun is worse than the OOB. The OOB has an isolation ratio of approximately $-45 \mathrm{~dB}$ at $1 \mathrm{~Hz}$, reducing to about $-85 \mathrm{~dB}$ at $10 \mathrm{~Hz}$. Thus, the static view does not give the complete picture of the gun's motion due to the vertical acceleration at the trunnions. The effect of the hull pitch rate disturbance, Fig. 3(b), for the OOB gun is very similar to the balanced, except that it is about $1 \mathrm{~dB}$ better up to $18 \mathrm{~Hz}$. This is because the inertia of the 


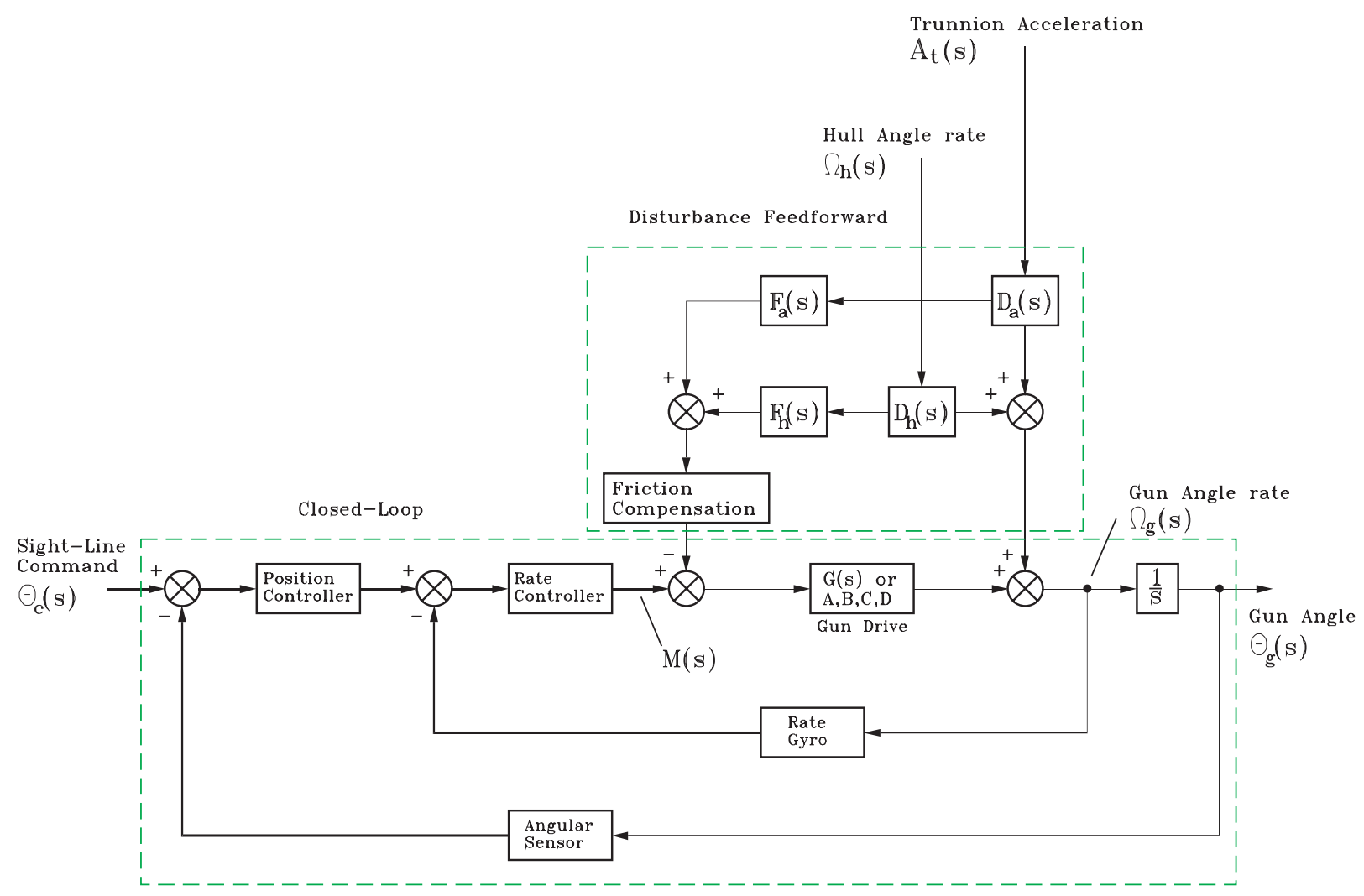

Fig. 4. Controller structure for the OOB and balanced weapon control systems.

OOB gun is greater than the balanced and thus resists the frictional and drive disturbance torques better.

\section{Weapon controller design}

This section examines the design of the closed-loop and disturbance feedforward controllers for the balanced and OOB weapon control systems. Only a brief discussion of the controller for the balanced gun is given here, a more detailed description is given in [3-5].

\subsection{Balanced gun}

To investigate the performance of the elevation model, a classical closed-loop controller was designed, which was based on the open-loop frequency responses. The form of the closed-loop controller is shown in the lower part of Fig. 4, and consists of an inner-loop breech rate controller and outer-loop breech position controller. The outer-loop controller is based on a traditional proportional plus integral structure, while the inner-loop has a proportional controller augmented with a notch and low pass filter. No attempt has been made to optimise the response of the closed-loop weapon control system. In addition to the closed-loop part, the controller is augmented with hull rate feedforward and non-linear friction compensation, this is to enhance the isolation of the gun from the hull [4].

\subsection{Out of balance gun}

The controller structure for the OOB gun is shown in Fig. 4. The controller has the same closed-loop and hull pitch rate feedforward components as the balanced. The proportional gain of the OOB case has been increased over the balanced by $8.3 \%$, so that they both have the same closed-loop bandwidths. In addition to this, the OOB controller has trunnion vertical acceleration feedforward. This is to help reduce the effect of the disturbance caused by the coupling between the vertical trunnion acceleration into the breech rotation.

\subsection{Feedforward controller design}

It can be shown [10] that for the trunnion vertical acceleration disturbance to have no effect on the gun, 

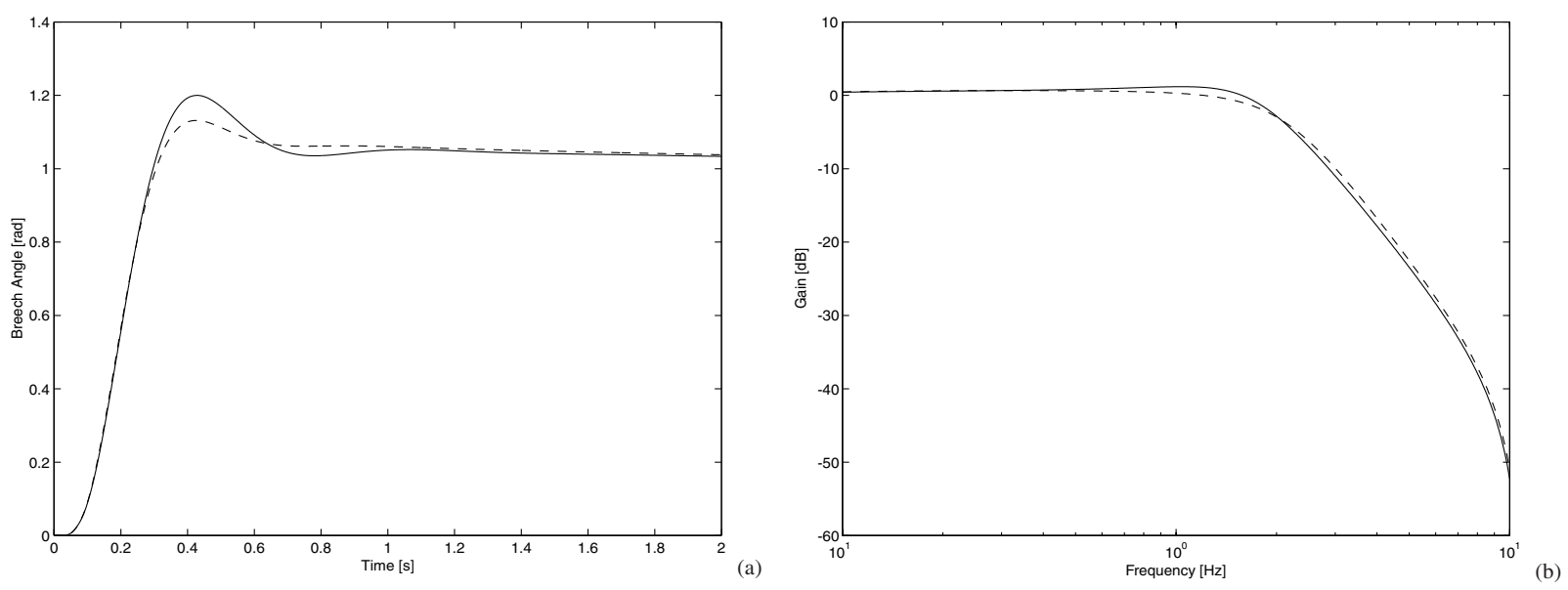

Fig. 5. Plot of closed-loop step (a) and frequency responses (b), with the OOB [solid] and balanced [dash] gun.

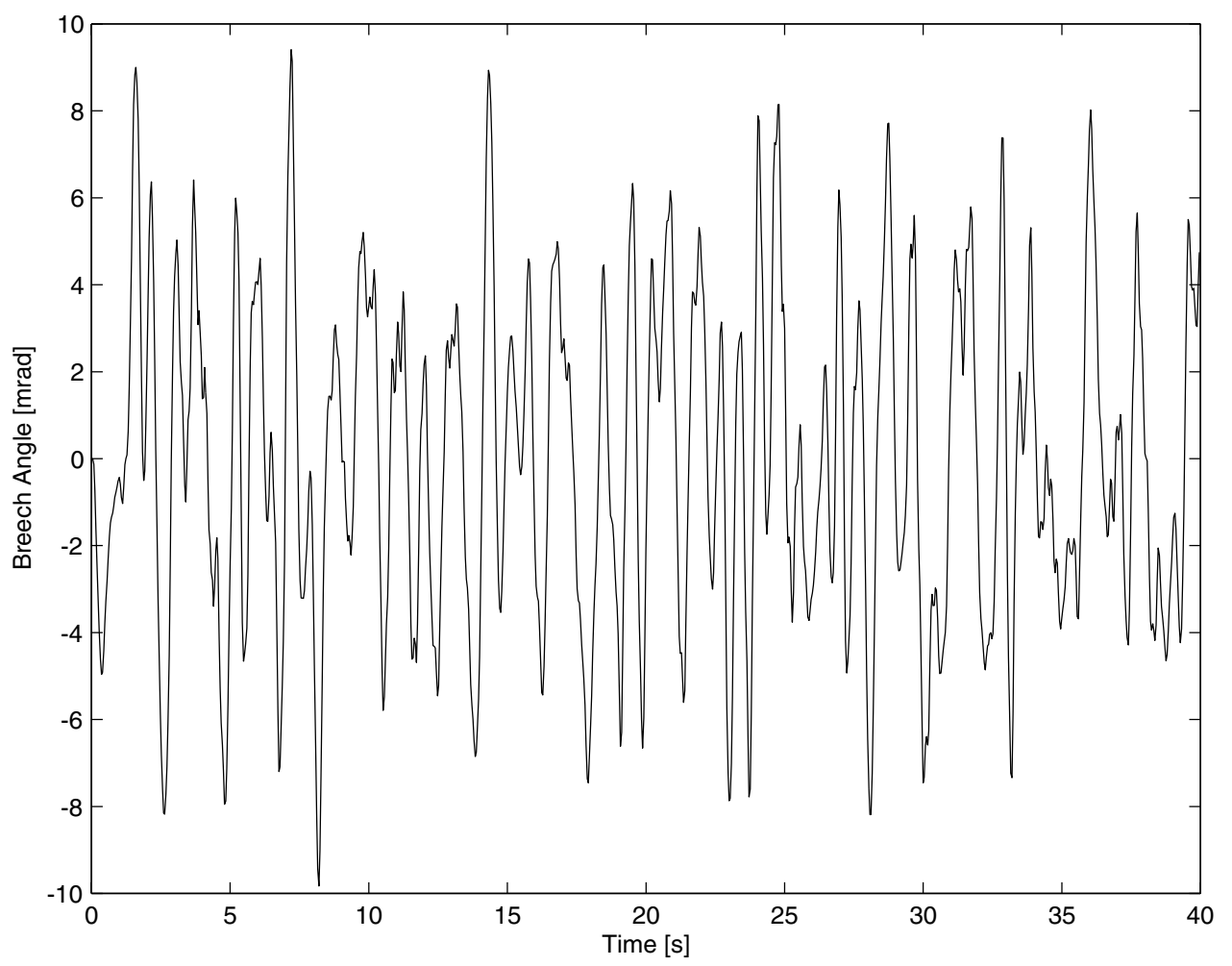

Fig. 6. Plot of the breech angle for the OOB without trunnion vertical acceleration feedforward.

the ideal feedforward controller $F_{a}(s)$ is given by:

$$
F_{a}(s)=\frac{D_{a}(s)}{G(s)}
$$

where $D_{a}(s)$ is the transfer function between the trunnion vertical acceleration $\ddot{y}_{t}$ and $\dot{\theta}_{1}$ and $G(s)$ is the transfer functions between $v_{i}$ and $\dot{\theta}_{1}$, see Fig. 4 .
Thus, if the feedforward controller is the ratio of the disturbance and system transfer functions, then the effect of the disturbance will be cancelled out. To simplify the transfer function for this feedforward controller design, the elevation model has been reduced to a single degree of freedom. This has been accomplished by removing the flexibility in the barrel and drive-line. 

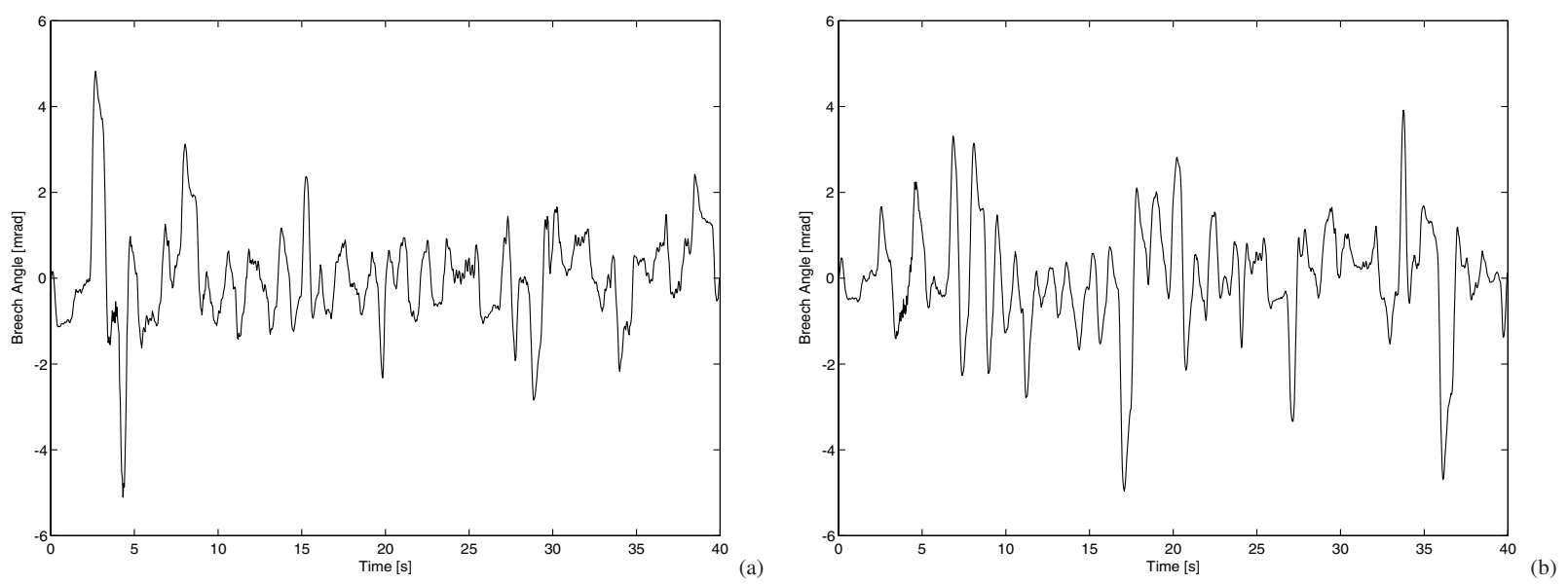

Fig. 7. Plot of the breech angle, with the balanced (a) and OOB (b) gun.

Thus the stiffness $k_{d}$ and $k_{12}$ are allowed to tend to infinity. The transfer function for this system, ignoring the hull pitch rate, is then given by:

$$
\Theta_{g}(s)=G(s) M(s)+D_{a}(s) A_{t}(s)
$$

where $M(s)$ and $A_{t}(s)$ are the command input and vertical trunnion acceleration respectively.

In this case:

$$
\begin{aligned}
& G(s)=\frac{K_{1} X_{t p} / R_{p}}{I_{e} s^{2}+c_{e} s} \\
& D_{a}=\frac{-\eta_{t} m_{t}}{I_{e} s^{2}+c_{e} s}
\end{aligned}
$$

where:

$$
\begin{gathered}
I_{e}=I_{g}+\left(X_{t p} / R_{p}\right)^{2} I_{d} \\
c_{e}=c_{g}+\left(X_{t p} / R_{p}\right)^{2} c_{d}
\end{gathered}
$$

The ideal feedforward transfer function is thus given by:

$$
F_{a}(s)=\frac{-\eta_{t} m_{t}}{K_{t} X_{t p} / R_{p}}
$$

For the trunnion vertical acceleration the ideal feedforward transfer function is a gain and can be implemented directly.

\subsection{Simulated closed-loop responses}

The closed-loop step and frequency responses for the balanced and OOB WCSs (linear models only) are shown in Fig. 5. The bandwidth, Fig. 5(b), for both cases are the same at approximately $2 \mathrm{~Hz}$, though the OOB case has a higher peak gain. The step responses, Fig. 5(a), show that the damping for the OOB situation is less than the balanced because of the greater overshoot.

\section{Simulation results and discussion}

In this investigation, simulation results have been obtained for the balanced and OOB weapon control systems on a MBT model crossing random terrain. The MBT vehicle model and terrain data have been taken from $[3,5]$. The simulation of the vehicle and WCSs was for 40 seconds.

A plot of breech motion for the OOB gun crossing the random terrain, without the trunnion vertical acceleration feedforward, is shown in Fig. 6. In this configuration the structure of the WCS is the same as the balanced. The rms breech angle, in this situation is $3.8 \mathrm{mrad}$.

A plot of the breech angle against time for the balanced and OOB gun, with trunnion vertical acceleration feedforward, is shown in Fig. 7. From examining this plot, it is not immediately obvious which of the weapon control systems is performing the best. The rms values for the balanced and OOB system are 1.14 and $1.27 \mathrm{mrad}$ respectively. Thus the OOB situation is $11.4 \%$ worse. The OOB gun without the trunnion acceleration feedforward, Fig. 6, is $3.80 \mathrm{mrad}$, which is almost $200 \%$ worse than with it. The motor torque, Fig. 8, shows that the OOB case has a greater amplitude and more higher frequency activity. There is an increase of $33.5 \%$ in the motor torque for the OOB over the balanced case. The rms power required by the OOB gun is $51.0 \mathrm{~W}$ and the balanced $44.0 \mathrm{~W}$, which is an increase of $15.9 \%$.

The largest increase in the OOB case is the rms motor torque demand at $33.5 \%$, while the rms breech angle and power have only small increases of $11.4 \%$ and $15.9 \%$ respectively. The results for the two cases are summarised in Table 1. 

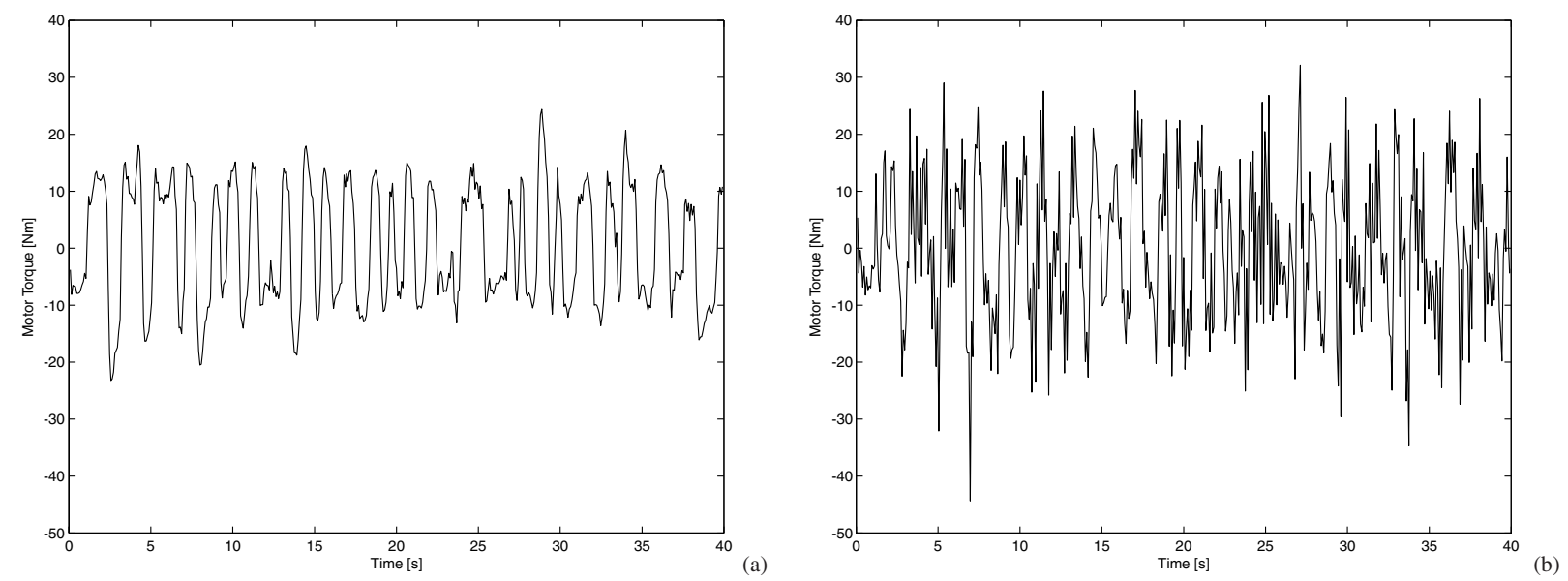

Fig. 8. Plot of the motor torque, balanced (a) and OOB (b).

Table 1

Comparison of balanced and OOB performance

\begin{tabular}{lccc}
\hline Parameter & \multicolumn{2}{c}{ rms } & \% Increase \\
\cline { 2 - 3 } & Balanced & OOB & \\
\hline Breech Angle [mrad] & 1.14 & 1.27 & 11.4 \\
Command Signal [v] & 1.94 & 2.59 & 33.5 \\
Motor Torque [Nm] & 10.55 & 14.09 & 33.5 \\
Power [W] & 44.0 & 51.0 & 15.9 \\
\hline
\end{tabular}

\section{Conclusion}

A comparison of an $\mathrm{OOB}$ and balanced gun in a MBT crossing random terrain has been presented. The controller for the OOB case has included a feedforward term derived from the vertical acceleration of the trunnions. The performance of the OOB controller without the feedforward term has been shown to be $200 \%$ worse, over the random terrain considered.

The results have shown conclusively that it is possible to stabilise an OOB gun on a moving platform, with only a small reduction in performance, an increase of $11.4 \% \mathrm{rms}$ breech angle motion and $15.9 \% \mathrm{rms}$ power.

\section{References}

[1] Rheinmetall Handbook on Weaponry, second English edition, Rheinmetal GmbH, 1992, pp. 385 and 386.
[2] H.D. Warwick, A guide to the design of main armament gun mountings for armoured fighting vehicles (U), DERA Chobham Lane Chertsey UK, Report No. 82019, pp. 61-66.

[3] D.J. Purdy, Modelling And Simulation Of A Weapon Control System For A Main Battle Tank, Proceedings Of The Eighth US Army Symposium On Gun Dynamics, 14-16 May 1996.

[4] D.J. Purdy, Main battle bank stabilisation ratio enhancement using hull rate feedforward, Journal of Battlefield Technology 1(2) (July 1998).

[5] D.J. Purdy, An Investigation Into The Modelling And Control Of Flexible Bodies, PhD. Thesis, Cranfield University (RMCS), England, 1994.

[6] D.K. Dholiwar, Development Of A Hybrid DistributedLumped Parameter Open Loop Model Of Elevation Axis For A Gun System, Proceedings Of The Seventh US Army Symposium On Gun Dynamics, 11-13 May 1993.

[7] P.T.L.M. Woerkom, On Fictitious Joints Modelling Of Manipulator Link Flexibility For The HERA Simulation Facility Pilot, National Aerospace Laboratory NLR The Netherlands, Report No. NLR TR 88086 U, 1988.

[8] D.A. Haessig and B. Friedland, On The Modeling And Simulation Of Friction, Transactions Of The ASME Journal Of Dynamic Systems, Measurement And Control 113 (Sep. 1991), 354-362.

[9] D.K. Miu, Physical interpretation of transfer function zeros for simple control systems with mechanical flexibilities, Journal of Dynamic Systems, Measurement and Control 113 (Sep. 1991).

[10] J.M. Maciejowski, Multivariable Feedback Design, AddisonWesley, 1989, pp. 10-13. 

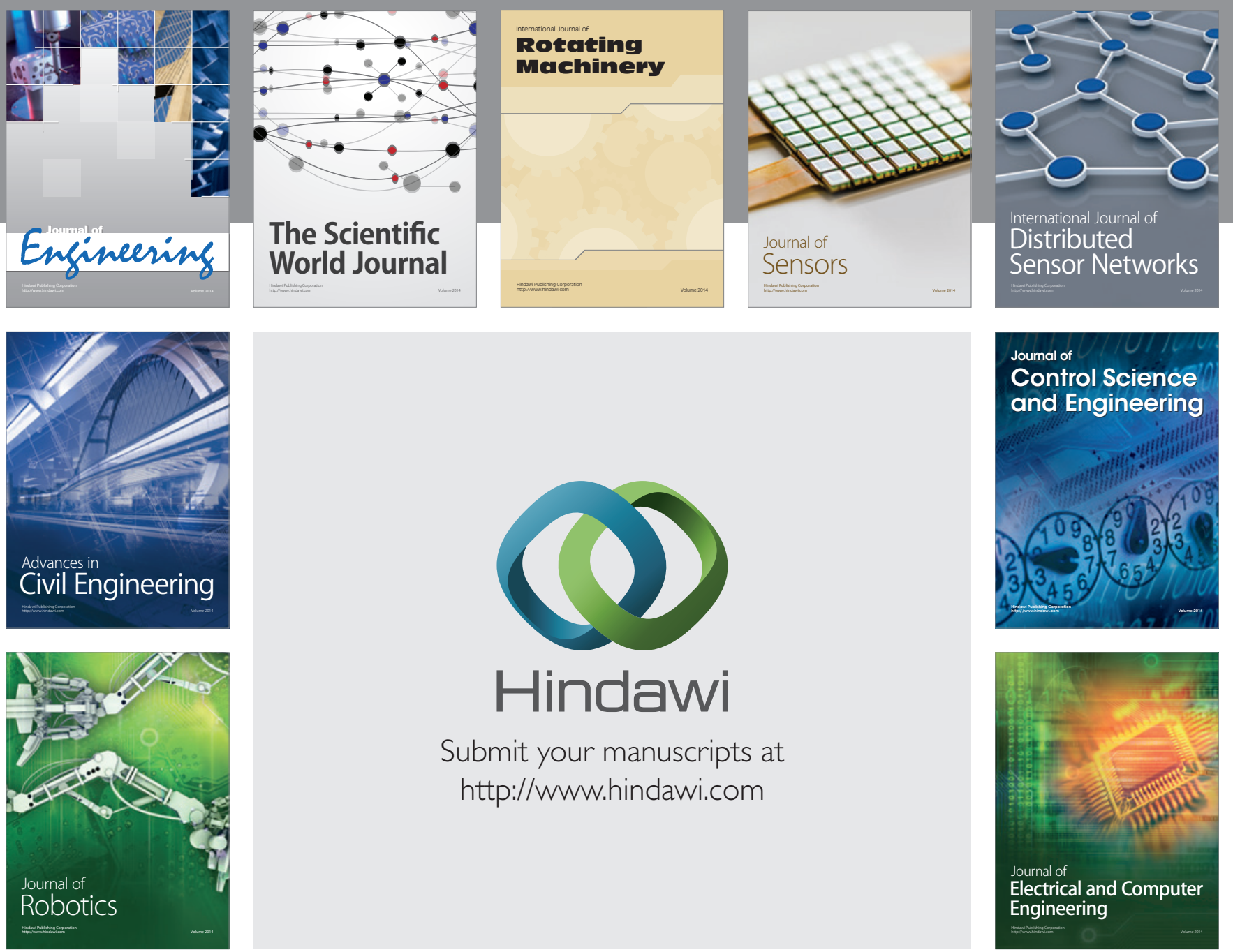

Submit your manuscripts at

http://www.hindawi.com
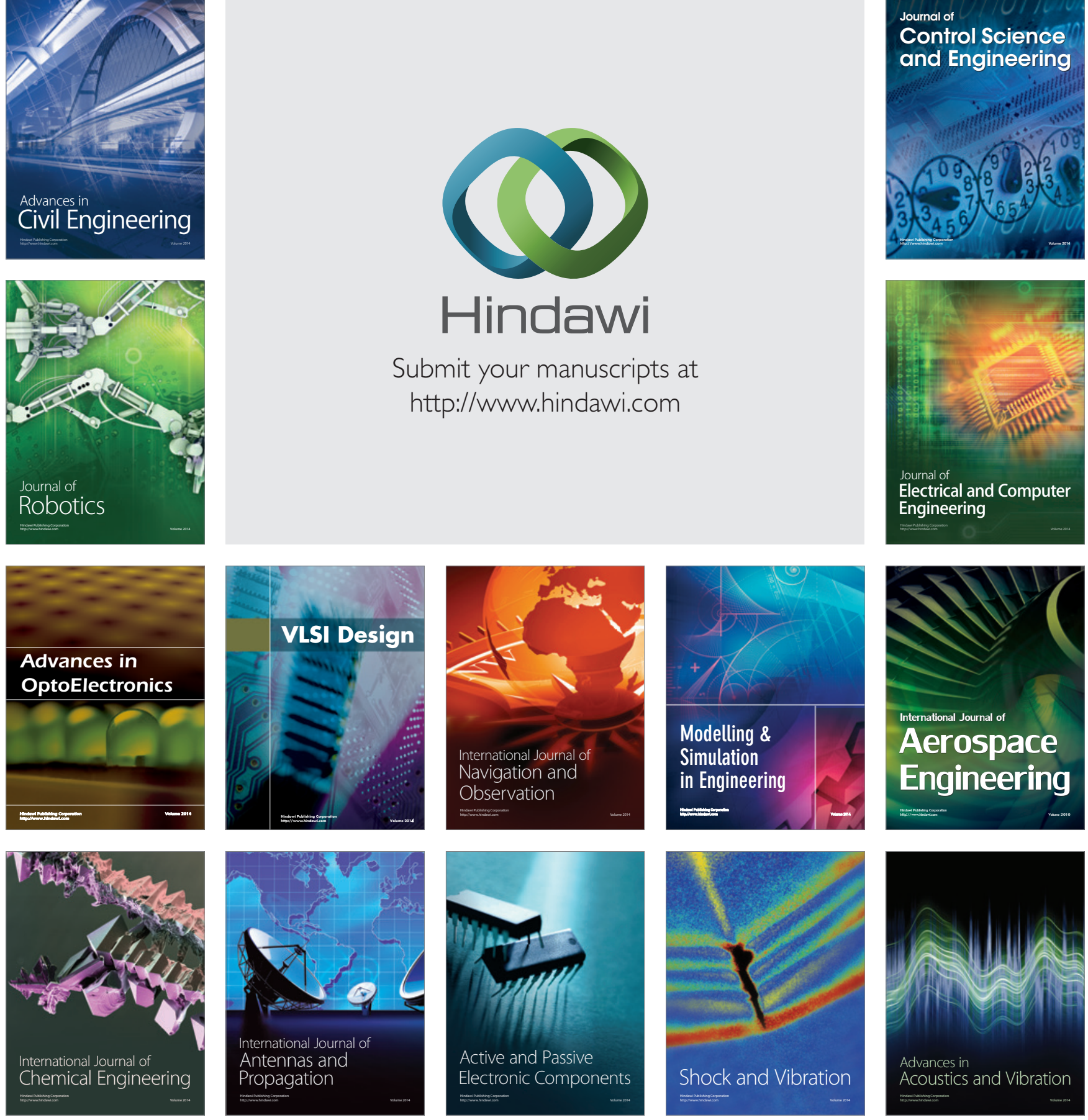